\title{
ALK-rearrangement neuroendocrine carcinoma of the lung: a comprehensive study of a rare case series and review of literature
}

This article was published in the following Dove Press journal:

OncoTargets and Therapy

\author{
Qiang Zheng ${ }^{1,2, *}$ \\ Mingjia Zheng ${ }^{1,2, *}$ \\ Yan Jin ${ }^{1,2}$ \\ Xuxia Shen ${ }^{1,2}$ \\ Ling Shan ${ }^{1,2}$ \\ Lei Shen ${ }^{1,2}$ \\ Yihua Sun ${ }^{2,3}$ \\ Haiquan Chen ${ }^{2,3}$ \\ Yuan $\mathrm{Li}^{1,2}$ \\ 'Department of Pathology, Fudan \\ University Shanghai Cancer Center, \\ Shanghai, China; ${ }^{2}$ Department of \\ Oncology, Shanghai Medical College \\ of Fudan University, Shanghai, China; \\ ${ }^{3}$ Department of Thoracic Surgery, \\ Fudan University Shanghai Cancer \\ Center, Shanghai, China \\ *These authors contributed equally \\ to this work
}

\begin{abstract}
Driver mutations involving tyrosine kinase receptors play crucial roles in the oncogenesis of lung adenocarcinoma. However, receptor tyrosine kinase mutations are extremely rare events in primary pulmonary neuroendocrine carcinoma (NEC), which is a molecular heterogeneous entity. In this study, we examined 4 cases of NEC with anaplastic lymphoma kinase $(A L K)$ rearrangement between 2008 and 2018 at our hospital. We comprehensively analyzed the carcinomas' clinicopathological features, genetic alterations, and response to ALK inhibitor. One case of atypical carcinoid tumor and 1 case of large cell NEC (LCNEC) achieved response to ALK inhibitor (crizotinib) treatment. One case of combined LCNEC with adenocarcinoma harboring KLC1-ALK (K9:A20) fusion genes was confirmed by NGS of both components, while only the LCNEC component presented RB1 mutation. Notably, tumor cells of different components exhibited different $A L K$-positive signal patterns by fluorescence in situ hybridization, which revealed isolated $3^{\prime}$ signals in the adenocarcinoma component but split signals in the LCNEC. As the largest case series study, our findings suggested that preliminary screening for $A L K$ rearrangement should also be considered in atypical carcinoid and high-grade NEC. Patients with $A L K$ rearrangement-positive NEC would benefit from ALK inhibitor intervention.
\end{abstract}

Keywords: anaplastic lymphoma kinase, driver mutation, neuroendocrine carcinoma, tumor evolution

\section{Introduction}

Driver mutations involving tyrosine kinases, such as epidermal growth factor receptor (EGFR) and anaplastic lymphoma kinase (ALK), have been shown to play an essential role in the pathogenesis of non-small cell lung cancer (NSCLC), especially in lung adenocarcinoma. ${ }^{1,2}$ Recently, genetic aberrations involving tyrosine kinase receptor have been potential targets of tyrosine kinase inhibitors (TKIs), EGFR-TKI, and ALK inhibitors are eminent representatives.

To the best of our knowledge, TP53/RB1 co-mutation is a significant and universal molecular event in the tumorigenesis of high-grade neuroendocrine carcinoma (NEC), including small cell lung carcinoma (SCLC) and large cell NEC (LCNEC). ${ }^{3,4}$ Recently, Rekhtman et al ${ }^{5,6}$ elucidated that LCNECs are molecularly heterogeneous, which could be further sub-classified into 3 major molecular subsets: small cell-like (characterized by $R B 1 / T P 53$ co-inactivation), non-small cell/adenocarcinoma-like (characterized by the lack of $R B 1 / T P 53$ co-mutation and frequent $K R A S$ and/or $S T K 11$ mutations), and a small subset of carcinoid-like (characterized by MEN1 mutations and low mutation burden) LCNEC. The molecular reclassification could help to elucidate the precise therapy and prognostic implications of this carcinoma.
Department of Pathology, Fudan

University Shanghai Cancer Center, 270

Dong An Road, Shanghai, 200032, China

Tel/fax +86 2I 64175590 ext 8317

Email lumoxuan2009@I63.com 
$A L K$ encodes anaplastic lymphoma kinase, a tyrosine kinase receptor that belongs to insulin receptor superfamily, activates downstream signaling pathway of cell survival, proliferation, and oncogenesis. In 2007, $A L K$ was first identified as a fusion gene involving the echinoderm microtubuleassociated protein-like 4 (EML4) gene in lung cancer. ${ }^{7}$ Since that finding, other fusion partners have been revealed with $A L K$, such as $K I F 5 B .{ }^{8}$ Clinically, the $E M L 4-A L K$ fusion gene has been detected in approximately $3 \%-7 \%$ of NSCLC cases, who are typically younger, non-smoking males. Histologically, $A L K$ fusion genes are associated with some histological characteristics in lung cancer, such as adenocarcinoma with mixed subtype, particularly in solid subtype with mucinous secretion. ${ }^{9}$ However, driver mutations involving $A L K$ fusions in pulmonary NEC are extremely rare. Few cases have been reported. ${ }^{10-19}$ A noteworthy phenomenon is that most of them were disseminated and high-grade lesions (3 atypical carcinoid tumors and 6 high-grade NECs). The detailed clinicopathological characters and outcome of anti-ALK targeted therapy are summarized in Table 1 . However, due to the rarity of these lesions, the clinical implication of $A L K$ rearrangement in NEC remains to be further elucidated. Therefore, in this study, we collected 4 cases of NEC with $A L K$ rearrangement between 2008 and 2018 at our hospital, including 2 cases of LCNEC, 1 case of combined LCNEC with adenocarcinoma, and 1 case of atypical carcinoid tumor. We comprehensively analyzed the clinicopathological features, genetic alterations, and response to ALK-TKI therapy to further investigate the clinical implication of $A L K$ rearrangement in pulmonary NECs.

\section{Patients and methods}

\section{Patients and samples}

Four patients with primary $A L K$ rearrangement-positive NEC were enrolled at our hospital. The clinicopathological information was obtained by reviewing their medical records. The pathological TNM (pTNM) stage was established according to the 8th edition of the American Joint Committee on Cancer/Union for International Cancer Control staging system. ${ }^{20}$ The histopathological classification of the lung cancers was according to the 2015 World Health Organization (WHO) classification. $^{21}$

\section{Analysis of $A L K$ gene rearrangement by fluorescence in situ hybridization (FISH)} FISH for $A L K$ (2p23) gene rearrangement was performed on formalin-fixed paraffin-embedded (FFPE) ( $3 \mu \mathrm{m}$ thick) sections according to a standard protocol using a commercially available $A L K$ break-apart probe (Abbott Molecular,

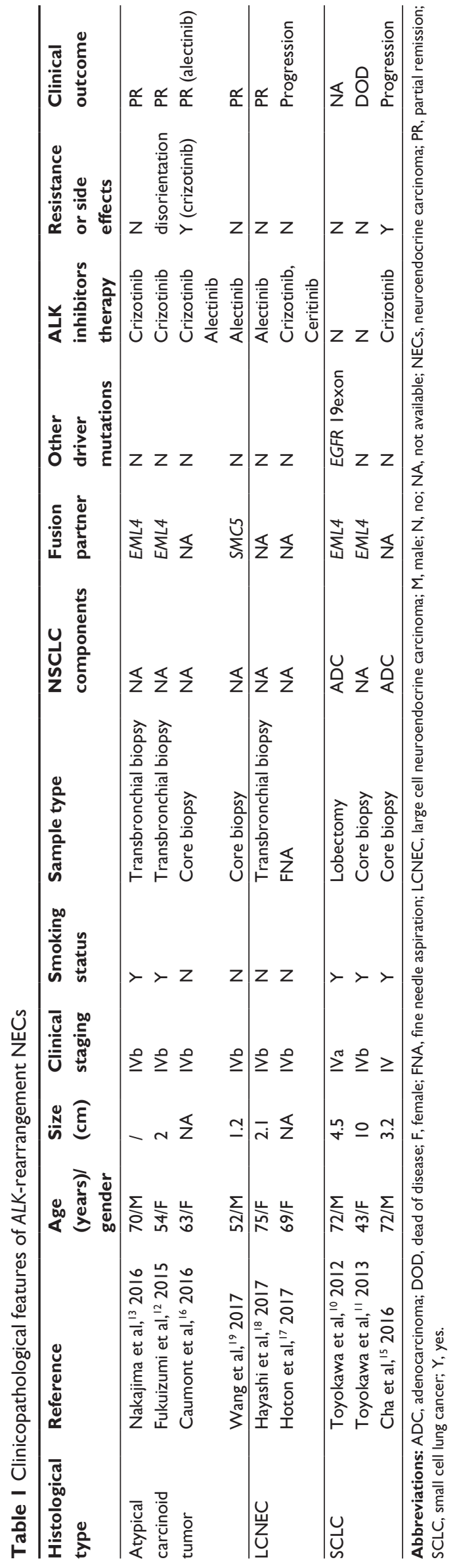


Des Plaines, IL, USA). Samples were considered to be FISH-positive if $>15 \%$ of the scored tumor cells had split or both $A L K 5^{\prime}$ and 3' probe signals or had isolated 3' signals. Slides were evaluated independently by 2 experts blind to the patients' history and histological findings.

\section{Immunohistochemical analysis}

Immunohistochemical analysis was performed on FFPE sections ( $4 \mu \mathrm{m}$ thick) using a fully automated system (Ventana Medical Systems, Tucson, AZ, USA). Slides were stained with antibodies against thyroid transcription factor-1 (TTF-1) (mouse monoclonal, clone 8G7G3/1; Ventana), Napsin-A (mouse monoclonal, clone KCG1-1; Abcam, Cambridge, MA, USA), CK7 (mouse monoclonal, clone SP52; Ventana), Synaptophysin (rabbit monoclonal, clone YE269; Abcam), CD56 (mouse monoclonal, clone ab5018; Abcam), chromogranin (rabbit polyclonal, clone ab15160; Abcam), and ALK (rabbit monoclonal, clone D5F3; Ventana).

\section{Next-generation sequencing (NGS)}

Genomic DNA was extracted from the FFPE tissue samples using the QIAamp DNA mini Kit(QIAGEN, Hilden, Germany), and targeted deep sequencing of mutational hotspots was conducted using a capture-based targeted sequencing panel (Burning Rock Biotech, Guangzhou, People's Republic of China), including all exons of 56 genes and selected introns in $A L K, R E T$, and ROS1 for the detection of translocation events. The library DNAs were prepared by amplifying the targeted regions using multiplex polymerase chain reaction (PCR), followed by adapter DNA ligation. Multiplexed sequencing was performed using the Illumina HiSeq 2,500 platform (Illumina, San Diego, CA, USA). Mutations and variants, including indels, substitutions, rearrangements, and amplifications, were identified using Illumina suite software.

\section{Ethics approval}

This study was approved by the institutional review board of Fudan University Shanghai Cancer Center. Four samples were enrolled from Fudan University Shanghai Cancer Center. All procedures performed in studies involving human participants were in accordance with the ethical standards of the institutional review board of Fudan University Shanghai Cancer Center. Written informed consent was provided by the patients regarding the publication of their case details and accompanying images.

\section{Results}

$A L K$ rearrangement was revealed in 4 samples by FISH or NGS. Histologically, 2 cases of LCNEC and 1 case of combined LCNEC with adenocarcinoma were identified in resection specimens. One case of atypical carcinoid tumor was identified by core biopsy. The clinicopathological features are summarized in Table 2.

\section{Case I}

A 44-year-old man with a 20-year smoking history presented with cough and back pain. Chest CT revealed an ill-defined lobulated mass in the left upper lobe. A lobectomy of the left upper lobe with dissection of mediastinal lymph nodes was performed. The greatest dimension of the resected tumor was $3.0 \mathrm{~cm}$. Lymphovascular invasion was identified, and 5 mediastinal lymph nodes (groups 5, 6, and 11) were positive for metastasis. Pleural invasion was not identified. Histologically, the tumor presented a solid growth pattern with nest and rosette architecture. Mitotic activity was prominent (15-20/10 high power fields [HPFs]) and multifocal necroses were easily identified. The lesion was positive for TTF-1, synaptophysin, chromogranin, and CD56, while it was negative for NapsinA. The tumor was pathologically diagnosed as LCNEC, and the primary pathological stage was pT1c. FISH for $A L K$ gene revealed split signals in $54 \%$ of tumor cells. The tumor was negative for other driver mutations. Four cycles of chemotherapy with cisplatin and pemetrexed were administered as the first-line regimen. Shortly after the chemotherapy, brain MRI revealed metastasis in the right occipital lobe. Therefore, the final clinical stage was IVb (cT1cN2M1c).

\section{Case 2}

A 64-year-old non-smoking woman underwent a routine chest CT, which showed a $4.5 \mathrm{~cm}$ lobulated mass in the right upper lobe and multiple enlarged mediastinal lymph nodes. Brain and abdominal MRI revealed multiple metastases in both cerebral hemispheres and the right adrenal gland. A core needle lung biopsy specimen from the right upper lobe showed that the bland tumor cells organized into trabeculae and solid nests with moderately abundant eosinophilic cytoplasm, several mitotic figures, and focal necrosis. The tumor cells were positive for TTF-1 and neuroendocrine markers, including synaptophysin, chromogranin, and CD56. The Ki-67 index was low (10\%) (Figure 1). Negativity of NapsinA helped rule out solid variant adenocarcinoma with neuroendocrine features. Thus, the diagnosis was neuroendocrine tumor, consistent with atypical carcinoid tumor, and the clinical stage was determined to be IVb (cT3cN2M1c). ALK immunostaining was performed and showed diffuse positivity, and subsequent FISH confirmed $A L K$ rearrangement. Crizotinib was administered as a first-line therapy. The lesions of the lung and adrenal gland shrunk dramatically 


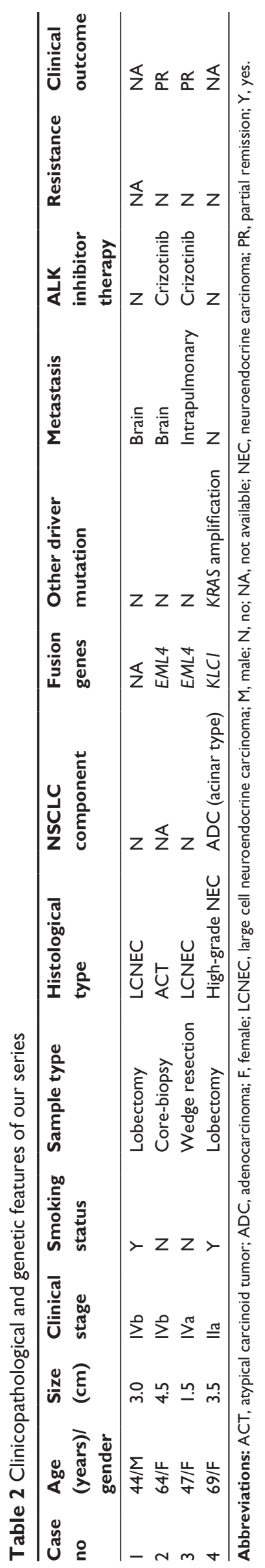

during treatment; however, the intracranial metastases still progressed 12 months later, and whole-brain irradiation was performed (DT $30 \mathrm{~Gy} / 10 \mathrm{f}$ ).

\section{Case 3}

A 47-year-old non-smoking female presented with chest pain and cough for 1 month. Chest CT revealed a $1.5 \mathrm{~cm}$ peripheral nodule in the right middle lobe, adjacent to the pleura. Mediastinal lymph nodes were enlarged and multiple satellite nodules were identified in the right lung. The patient underwent a wedge resection of right middle lobe. Histologically, the tumor cells were arranged in solid nests with multiple necroses. The tumor cells showed prominent atypia and mitotic activity (20/10 HPFs). Lymphovascular invasion was frequently seen. This lesion showed TTF-1+/ NapsinA-phenotype. The neuroendocrine markers, including CD56, Syn, and CgA were diffuse and strong positive in immunohistochemical staining. This tumor was pathologically consistent with LCNEC. The clinical stage was IVa (cT1cN1M1a). ALK immunostaining was performed and showed diffuse and strong positivity. Subsequent NGS of circulation tumor DNA confirmed $E M L 4-A L K$ fusion. Other driver mutations were not detected. Treatment with crizotinib was administered. Ten months later, radiological evaluation revealed a rapid regression of all known lesions.

\section{Case 4}

A 69-year-old female former smoker of 700 pack-years underwent a routine chest $\mathrm{CT}$, which demonstrated an illdefined mass in the left upper lobe of the lung. The mass was assumed to be primary lung cancer, and left upper lobectomy with lymphadenectomy was performed. The cut surface revealed a gray-white solid mass with a maximum diameter of $3.5 \mathrm{~cm}$. Pathologically, the tumor was composed of 2 separate components. The central solid area, which consisted of $60 \%$ of the entire tumor mass, was a highgrade NEC, and the surrounding area was adenocarcinoma with acinar and papillary components. Both components showed immunoreactivity to TTF-1, whereas synaptophysin, chromogranin, and CD56 were positive only in the NEC component. Additionally, NapsinA expression was only detected in adenocarcinoma area and consistently absent in the NEC area. The pathological stage was determined to be pT2c. Each of the components was separately examined for driver gene mutations of lung cancer by FISH and NGS. $A L K$ FISH showed $60 \%$ rearrangement in both components. Interestingly, isolated $A L K 3^{\prime}$ signals were detected only in the adenocarcinoma component, whereas split signals were 

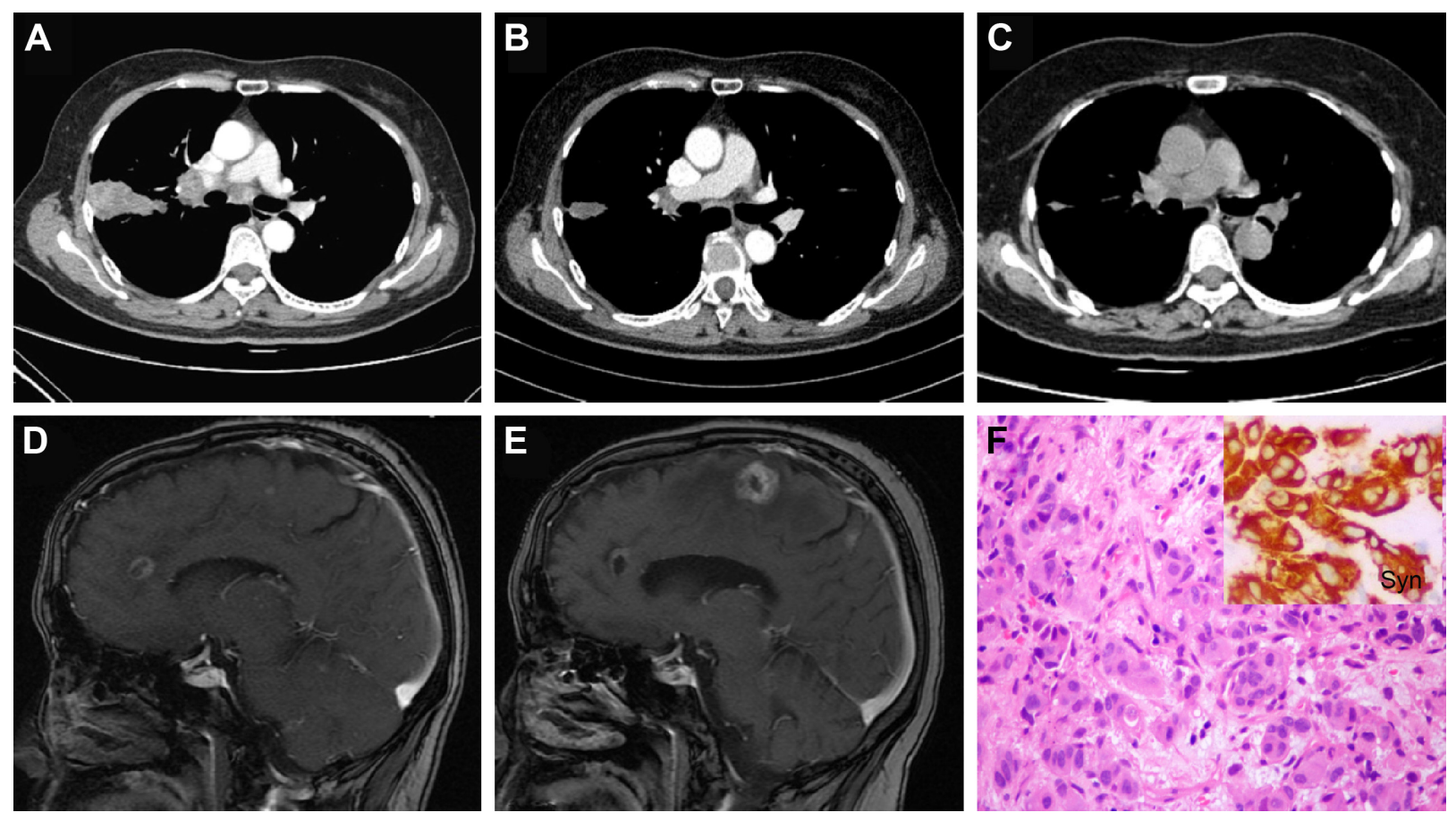

Figure I Contrast CT scan showed a $4.5 \mathrm{~cm}$ lobulated mass in the right upper lobe and multiple enlarged mediastinal lymph nodes (A). The lung lesions regressed dramatically with administration of crizotinib for I month (B) and 12 months (C). Brain MRI revealed multiple metastases in the cerebral hemispheres and cerebellum bilaterally (not shown) (D). However, the intracranial lesions progressed 12 months later (E). A core needle lung biopsy specimen revealed trabecular and solid nests with moderately abundant eosinophilic cytoplasm, and was positive for neuroendocrine markers (F, H\&E ×200).

found only in the NEC component. Both components showed diffuse and strong immunoreactivity to ALK protein by highsensitivity immunohistochemistry (Figure 2). KLC1-ALK (K9:A20) fusion gene was identified in both components, but RB1 mutation and KRAS amplification were only detected in the NEC component.

\section{Discussion}

Primary NECs, including typical carcinoid, atypical carcinoid, SCLC, and LCNEC were identified by pathological identification of the neuroendocrine morphology and immunohistochemical profile. High-grade NECs are highly aggressive NEC in the lung, for which the diagnosis and first-line treatment are still problematic for pathologists and clinicians. According to the 2015 WHO classification of lung cancer, pulmonary LCNEC is defined by the following features: neuroendocrine morphology (nesting, peripheral palisading, and rosettes), expression of neuroendocrine immunohistochemistry markers, and mitotic activity $>10$ mitoses/10 HPFs $\left(2 \mathrm{~mm}^{2}\right) .{ }^{21}$ Recently, Rekhtman et $\mathrm{al}^{6}$ concluded that LCNECs are heterogeneous with regard to molecular genetics and could be separated into 3 molecular subtypes: small cell-like, non-small cell/adenocarcinoma-like, and carcinoid-like. The molecular subtypes could predict the responses to standard chemotherapy regimens, which is meaningful for guiding chemotherapy and prognosis prediction. According to Derks et al, ${ }^{22}$ LCNEC tumors that harbor a wild-type and/or express $\mathrm{RB} 1$ protein have prolonged overall survival when treated with NSCLC chemotherapy compared with SCLC chemotherapy. By definition, non-small cell/adenocarcinoma-like LCNEC is different from adenocarcinoma with neuroendocrine differentiation. However, the 2 entities may actually be a continuous lineage.

Driver mutations involving $A L K$ gene (mainly rearrangement) are extremely rare in primary pulmonary NEC. According to previous files and this study, most NECs with $A L K$ translocation are high grade and in advanced stage, which closely correlate with poorer prognosis. $A L K$ rearranged NECs may be a specific molecular subtype with aggressive behavior. Due to the limitation of traditional regimen, targeted therapy may benefit this subset of patients. Nakamura et $\mathrm{al}^{23}$ performed high-sensitivity ALK immunostaining on 227 pulmonary NEC tissue microarrays, which showed focal positivity with heterogeneous intensity in 2 SCLCs and 1 LCNEC. However, neither $A L K$ rearrangement nor amplification was observed by FISH, and no somatic mutation was detected in 3 ALK-positive NECs. Thus, the aberrant expression of ALK is a potential pitfall 

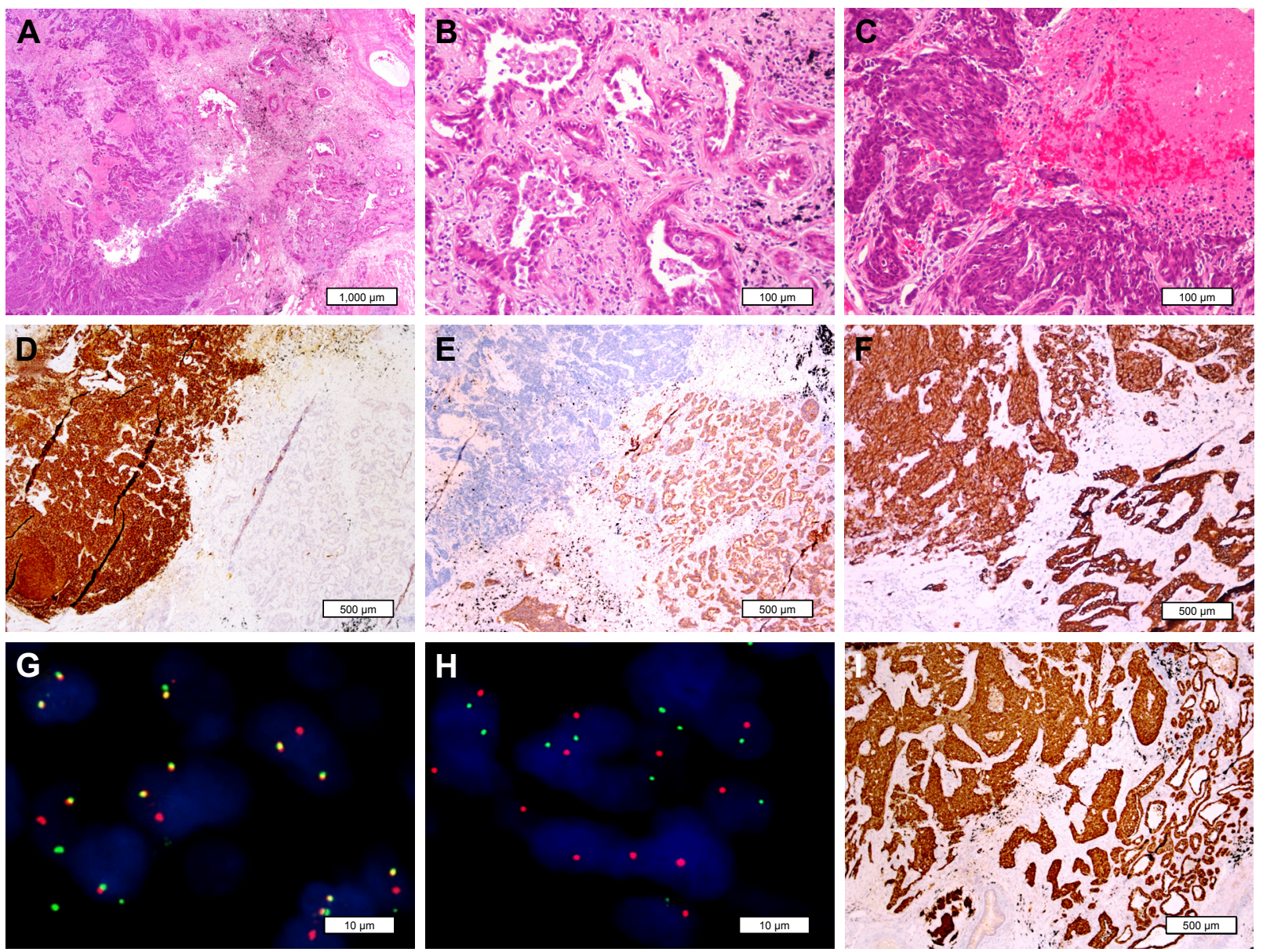

Figure 2 At low power, the neuroendocrine carcinoma (right) and adenocarcinoma component (left) were clearly separated (A, H\&E $\times 20)$. At high-power magnification, necrosis and mitotic figures were frequently seen in adenocarcinoma (B) and neuroendocrine carcinoma (C, H\&E $\times 400)$. Synaptophysin was positive only in the NEC component $(\mathbf{D}, \mathrm{IHC} \times 40)$. NapsinA was only positive in the adenocarcinoma area $(\mathbf{E}, \mathrm{IHC} \times 40)$. Both components showed immunoreactivity to $\mathrm{CK} 7(\mathbf{F}$, IHC $\times 40)$. FISH for $A L K$ gene revealed that split signals and isolated $3^{\prime}$ signals were observed in adenocarcinoma and neuroendocrine carcinoma components, respectively ( $\mathbf{G}$ and $\left.\mathbf{H}\right)$. ALK rearrangement was also confirmed by ALK (D5F3) immunostaining $(\mathrm{I}, \mathrm{IHC} \times 40)$.

Abbreviations: ALK, anaplastic lymphoma kinase; FISH, fluorescence in situ hybridization; IHC, immunohistochemistry.

in the molecular diagnosis of lung NECs. Between 2008 and 2018, 70 cases of carcinoid tumors and 76 LCNECs were diagnosed in resection specimen in our institution. Since 2014, we have routinely screened the status of $A L K$ in all NSCLC by high-sensitivity immunohistochemistry (IHC), regardless of histologic type. However, only 4 cases of NEC with $A L K$-rearrangement were identified. The frequency $(2.7 \%)$ is slightly lower than that in adenocarcinoma (3\%-5\%). As the largest case series study of $A L K$-rearranged NECs, the 4 cases were all confirmed by FISH or NGS. Concerning targeted therapy, the presence of $A L K$ fusion genes should be validated by FISH, reverse transcription PCR, or NGS, especially when the expression of ALK protein is focal or heterogeneous by IHC. Therefore, the combination of FISH and IHC is recommended to accurately identify the patients suitable for targeted therapy.
The fourth case in our study consisted of 2 completely different histopathological areas, which showed the morphology and immunophenotypes of both LCNEC and adenocarcinoma. Admittedly, KLC1-ALK fusion genes were identified in both elements, whereas only the NEC component harbored $R B 1$ mutation. Furthermore, different $A L K$-positive signal patterns were ascertained in the $2 \mathrm{com}-$ ponents by FISH, which phenomenon has not been previously reported in the literature. In keeping with prior studies, isolated and attenuated $A L K 3^{\prime}$ signal is associated with novel translocation partners, such as $C E B P \zeta .{ }^{24}$ However, because the 2 histopathological components shared the same fusion of KLC1-ALK, the mechanism and significance of isolated $A L K 3^{\prime}$ signal remain to be further elucidated. Therefore, we hypothesized that the transformation of neuroendocrine phenotype on the basis of adenocarcinoma is closely related 
to the loss of $R B 1$, the inactivation of which seems to be a key event leading to the transformation of high-grade NEC.,25 This phenomenon likely reflects the evolutionary process and intratumor heterogeneity of lung cancer. Thus, combined NEC with adenocarcinoma and adenocarcinoma with neuroendocrine differentiation may be actually a dynamic evolutionary process of adenocarcinoma harboring the same driver mutations.

SCLC or LCNEC transformation after EGFR-TKI treatment is one of the mechanisms of TKI resistance in NSCLC. ${ }^{26}$ This observation is attributed to the selective pressure during the TKI treatment, which upregulates related signal pathways and transforms the morphology and phenotype of the tumor. However, unlike EGFR-TKIs, this phenomenon has rarely been reported as an acquired resistance mechanism in $A L K$ rearrangement-positive patients. In prior studies, most of the patients with ALK-rearranged NEC can be successfully treated with ALK inhibitor, but several cases showed resistance or progression. Therefore, some authors hypothesized that the acquisition of neuroendocrine phenotype is also one of the reasons for the resistance of ALK inhibitors. ${ }^{15-17}$ One case also reported that sarcomatoid transformation of $A L K$-rearranged adenocarcinoma with $A L K$ amplification was resistant to ALK inhibitors. ${ }^{27}$ However, in our samples, the morphological and phenotypic transformation occurred without prior anti-ALK treatment, thus the aforementioned mechanism does not apply to our cases. In our study, 2 patients have been taking ALK inhibitor and 1 achieved dramatic response at the end of follow-up. In contrast, for another patient, although the primary lesion remained stable, the intracranial metastases still progressed. This phenomenon is partially due to the poor penetration of the blood-brain barrier by crizotinib, which limits its activity on brain metastases. The second- and third-generation ALK inhibitors, ceritinib, alectinib, brigatinib, and lorlatinib, show higher central nervous system (CNS) penetration and better response in pre-treated patients. ${ }^{28-32}$ Thus, we suggest that second- or third-generation ALK inhibitors would be a better choice for $A L K$-rearranged NSCLC with diffuse CNS involvement.

\section{Conclusion}

According to our experience and previous reports, although $A L K$ rearrangement is an extremely rare event in NEC, this phenomenon indicated that driver mutations tests, including ALK rearrangement, should also be recommended in atypical carcinoid tumors and high-grade NECs. High-sensitivity ALK immunostaining is sensitive and specific for preliminary screening. This subset of NEC harboring $A L K$ rearrangement would also benefit from ALK inhibitor therapy.

\section{Acknowledgment}

This study was supported by the National Natural Science Foundation of China (grant no. 81472173).

\section{Disclosure}

The authors report no conflicts of interest in this work.

\section{References}

1. Pao W, Girard N. New driver mutations in non-small-cell lung cancer. Lancet Oncol. 2011;12(2):175-180.

2. Zhou JX, Yang H, Deng Q, et al. Oncogenic driver mutations in patients with non-small-cell lung cancer at various clinical stages. Ann Oncol. 2013;24(5):1319-1325.

3. Miyoshi T, Umemura S, Matsumura Y, et al. Genomic profiling of large-cell neuroendocrine carcinoma of the lung. Clin Cancer Res. 2017; 23(3):757-765.

4. Peifer M, Fernández-Cuesta L, Sos ML, et al. Integrative genome analyses identify key somatic driver mutations of small-cell lung cancer. Nat Genet. 2012;44(10):1104-1110.

5. Rekhtman N, Pietanza MC, Hellmann MD, et al. Next-generation sequencing of pulmonary large cell neuroendocrine carcinoma reveals small cell carcinoma-like and non-small cell carcinoma-like subsets. Clin Cancer Res. 2016;22(14):3618-3629.

6. Rekhtman N, Pietanza CM, Sabari J, et al. Pulmonary large cell neuroendocrine carcinoma with adenocarcinoma-like features: napsin A expression and genomic alterations. Mod Pathol. 2018;31(1):111-121.

7. Soda M, Choi YL, Enomoto M, et al. Identification of the transforming EML4-ALK fusion gene in non-small-cell lung cancer. Nature. 2007; 448(7153):561-566

8. Takeuchi K, Choi YL, Togashi Y, et al. KIF5B-ALK, a novel fusion oncokinase identified by an immunohistochemistry-based diagnostic system for ALK-positive lung cancer. Clin Cancer Res. 2009;15(9): 3143-3149.

9. Inamura K, Takeuchi K, Togashi Y, et al. EML4-ALK fusion is linked to histological characteristics in a subset of lung cancers. $J$ Thorac Oncol. 2008;3(1):13-17.

10. Toyokawa G, Taguchi K, Ohba T, et al. First case of combined small-cell lung cancer with adenocarcinoma harboring EML4-ALK fusion and an exon 19 EGFR mutation in each histological component. $J$ Thorac Oncol. 2012;7(12):e39-e41.

11. Toyokawa G, Takenoyama M, Taguchi K, et al. An extremely rare case of small-cell lung cancer harboring variant 2 of the EML4-ALK fusion gene. Lung Cancer. 2013;81(3):487-490.

12. Fukuizumi A, Akagi K, Sakai H. A case of atypical carcinoid: harboring variant 3a/b EML4-ALK rearrangement. J Thorac Oncol. 2015; 10(10):e104-e106.

13. Nakajima M, Uchiyama N, Shigemasa R, Matsumura T, Matsuoka R, Nomura A. Atypical carcinoid tumor with anaplastic lymphoma kinase (ALK) rearrangement successfully treated by an ALK inhibitor. Intern Med. 2016;55(21):3151-3153.

14. Fujita S, Masago K, Katakami N, Yatabe Y. Transformation to SCLC after treatment with the ALK inhibitor alectinib. J Thorac Oncol. 2016;11(6):e67-e72.

15. Cha YJ, Cho BC, Kim HR, Lee HJ, Shim HS. A case of ALK-rearranged adenocarcinoma with small cell carcinoma-like transformation and resistance to crizotinib. J Thorac Oncol. 2016;11(5):e55-e58.

16. Caumont C, Veillon R, Gros A, Laharanne E, Bégueret H, Merlio JP. Neuroendocrine phenotype as an acquired resistance mechanism in ALKrearranged lung adenocarcinoma. Lung Cancer. 2016;92(92):15-18. 
17. Hoton D, Humblet Y, Libbrecht L. Phenotypic variation of an ALKpositive large-cell neuroendocrine lung carcinoma with carcinoid morphology during treatment with ALK inhibitors. Histopathology. 2018;72(4):707-710.

18. Hayashi N, Fujita A, Saikai T. Large Cell Neuroendocrine Carcinoma Harboring an Anaplastic Lymphoma Kinase (ALK) Rearrangement with Response to Alectinib. Intern Med. 2018;57(5):713-716.

19. Wang VE, Young L, Ali S, et al. A case of metastatic atypical neuroendocrine tumor with $A L K$ translocation and diffuse brain metastases. Oncologist. 2017;22(7):768-773.

20. Amin MB, Edge SB, Greene FL. AJCC Cancer Staging Manual. Chicago, IL: Springer International Publishing 2017.

21. Travis WD, Brambilla E, Burke AP, Marx A, Nicholson AG. editors. WHO Classification of Tumours of Lung, Pleura, Thymus and Heart. Lyon: International Agency for Research on Cancer; 2015.

22. Derks JL, Leblay N, Thunnissen E, et al. Molecular subtypes of pulmonary large-cell neuroendocrine carcinoma predict chemotherapy treatment outcome. Clin Cancer Res. 2018;24(1):33-42.

23. Nakamura H, Tsuta K, Yoshida A, et al. Aberrant anaplastic lymphoma kinase expression in high-grade pulmonary neuroendocrine carcinoma. J Clin Pathol. 2013;66(8):705-707.

24. Li W, Zhang J, Guo L, Chuai S, Shan L, Ying J. Combinational analysis of FISH and immunohistochemistry reveals rare genomic events in ALK fusion patterns in NSCLC that responds to crizotinib treatment. J Thorac Oncol. 2017;12(1):94-101.
25. George J, Lim JS, Jang SJ, et al. Comprehensive genomic profiles of small cell lung cancer. Nature. 2015;524(7563):47-53.

26. Oser MG, Niederst MJ, Sequist LV, Engelman JA. Transformation from non-small-cell lung cancer to small-cell lung cancer: molecular drivers and cells of origin. Lancet Oncol. 2015;16(4):e165-e172.

27. Kobayashi Y, Sakao Y, Ito S, et al. Transformation to sarcomatoid carcinoma in ALK-rearranged adenocarcinoma, which developed acquired resistance to crizotinib and received subsequent chemotherapies. J Thorac Oncol. 2013;8(8):e75-e78.

28. Peters S, Camidge DR, Shaw AT, et al. Alectinib versus crizotinib in untreated ALK-positive non-small-cell lung cancer. $N$ Engl $J$ Med. 2017;377(9):829-838.

29. Shaw AT, Felip E, Bauer TM, et al. Lorlatinib in non-small-cell lung cancer with ALK or ROS1 rearrangement: an international, multicentre, open-label, single-arm first-in-man phase 1 trial. Lancet Oncol. 2017; 18(12):1590-1599.

30. Zhang S, Anjum R, Squillace R, et al. The Potent ALK inhibitor Brigatinib (AP26113) overcomes mechanisms of resistance to first- and second-generation ALK inhibitors in preclinical models. Clin Cancer Res. 2016;22(22):5527-5538.

31. Friboulet L, Li N, Katayama R, et al. The ALK inhibitor ceritinib overcomes crizotinib resistance in non-small cell lung cancer. Cancer Discov. 2014;4(6):662-673.

32. Shaw AT, Kim DW, Mehra R, et al. Ceritinib in ALK-rearranged non-small-cell lung cancer. N Engl J Med. 2014;370(13):1189-1197.
OncoTargets and Therapy

\section{Publish your work in this journal}

OncoTargets and Therapy is an international, peer-reviewed, open access journal focusing on the pathological basis of all cancers, potential targets for therapy and treatment protocols employed to improve the management of cancer patients. The journal also focuses on the impact of management programs and new therapeutic agents and protocols on

\section{Dovepress}

patient perspectives such as quality of life, adherence and satisfaction. The manuscript management system is completely online and includes a very quick and fair peer-review system, which is all easy to use. Visit http://www.dovepress.com/testimonials.php to read real quotes from published authors. 